Volume 9, No.4, July - August 2020

International Journal of Advanced Trends in Computer Science and Engineering

Available Online at http://www.warse.org/IJATCSE/static/pdf/file/ijatcse111942020.pdf

https://doi.org/10.30534/ijatcse/2020/111942020

\title{
A Paradigm Shift towards On-Premise Modern Data Center Infrastructure for Agility and Scalability in Resource Provisioning
}

\author{
Bharani Dharan $\mathbf{G}^{\mathbf{1}}$, Dr.Jayalakshmi $\mathrm{S}^{\mathbf{2}}$ \\ ${ }^{1}$ Research Scholar, Computer Science, VISTAS, Pallavaram, Chennai, mailto.bharanidharang@ rediffmail.com \\ ${ }^{2}$ Professor, Dept. Of Computer Applications, VISTAS, Pallavaram, Chennai, jai.scs@ velsuniv.ac.in
}

\section{ABSTRACT}

In this Modern Era, Datacenters are the backbone of today's enterprise businesses. To expand their business growth, an organization should first identify whether they are going to stay with on-premises or cloud infrastructure. In this paper we are going to explore the evolution towards on-premises Modern Data Centre (DC) from the basic classical, "siloed" traditional infrastructure. The old traditional data centre relies on more hardware and physical servers, needs more individual team to maintain, consumes more electricity, incurs more cost on buying machines for extra workloads when company grows. Maintaining the data securely is also a crucial task in siloed infrastructure because it needs more configuration and administration task which may lead to error-prone. The Next level of converged infrastructure in which the configurations are of pre-defined bundled templates which cannot be scaled further. The one more level-up the existing on-premise hyper-converged infrastructure in which CPU and storage will be coupled in one plane and network is in another plane and here when scalability is needed extra nodes to be created completely again is an additional overhead. In this paper the proposed Composable Infrastructure which supports all kinds of traditional and modern workloads with fluid pool of independent resource provisioning can be done intelligently through predictive unified API with more scalability, High availability and agility by means of the technologies like virtualization and containerization like the power of cloud infrastructure and services. Moreover, the software industry is highly fluid in this present decade to adapt the old software model to fit with evolving containerized Micro-services based applications which requires greater scalability and fast deployment. Hence the evolved on-premises composable modern infrastructure which is more dynamic in provisioning the resources with unlimited scaling is compared with other existing infrastructures with various parameters like Energy efficiency, High Availability, Cost and Agility.

Key words: Agility, Containerization, Hyper-Converged Infrastructure, Modern Composable Infrastructure,
On-Premise Infrastructure, Scalability, Siloed, Virtualization,

\section{INTRODUCTION}

In this present decade the large enterprises, Information Technology (IT), health care sectors, educational institutions and other medium and large firms produces huge amount of big data and thereby On-Premises Modern Software-Defined Data Centers (SDDC) came into a role to process, store and manage those data efficiently with intelligence with less carbon footprints. Companies that want to compete in today's fast changing digital environment should focus on innovative technologies for organizational business agility based on high volumes of real time data. The traditional old on-premise data centre relies heavily on hardware and physical IT infrastructure such as server, Network, storage, Firewall is hosted in within their organization with local software. Traditional On-premise DC is based on do-it-yourself approach where the compute, storage and network are maintained internally by the enterprises for secure deployment capabilities [1]. A Traditional infrastructure has built with individual units with application servers, separate Storage, network switches that are linked together and these each units should be configured individually which requires dedicated team of IT experts and each of them specialized in various field. Sometimes in on-premise traditional DC each unit will be from different vendor and their service warranty and support are maintained individually. So called Traditional DC is siloed because it makes use of various generations of software and hardware from old mainframes to rack-scale design architecture solutions. In the on-premises traditional DC when equipment failure happen it cannot be replaced instantly because of rigidness between the hardware and components from various manufacturers. So procuring the new hardware from various vendors may consume time and cost to replace the failed component [2]. Traditional on-premise data centre infrastructure also suffers from standards, lack of interoperatabilty, more hardware dependency and omnipresent management solutions [3]. In on-premises Traditional DC, Enterprises invests costs in more hardware like servers, storage and network equipment's 
to meet specific application needs required by their organization employees to process their workloads and keep them secure within their datacenter privately. Sometimes in legacy siloed infrastructure for managing the different workloads, over-provisioning may happen which increases the Capital Expenditures (CAPEX) also. Traditional infrastructure within on-premise is very expensive and not very flexible. In on-premise data center there is a requirement of standardizing hardware and software components is necessary with Software-Defined (SD) solutions for evolution of client-server architecture and particularly in terms of "cloud like" or cloud-par rapid service provisioning. The traditional on-premise data centers depend on monolithic server interconnection with fragmented resource usage leads to power incompetence and increases the Total Cost of Ownership (TCO) [4]. The limitation in the on-premises Traditional DC is of static design and usage limits of hardware resource services cannot be utilized efficiently. To overcome this limitation, the other on-premise infrastructures which is of virtualized datacenters like SDDC 2.0, SDDC 3.0 and SDDC 4.0 has evolved to handle the existing homogenous and other heterogeneous modern workloads with unlimited scalability and flexibility [5] [6]. In the below diagram the transition or evolution process of various on-premise datacenter architecture has shown in figure 1 [7].

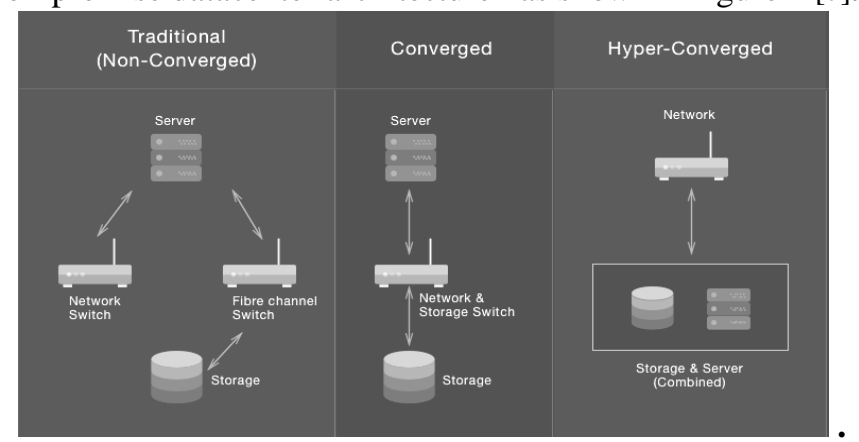

Figure 1: Transition towards on-premises Software-Defined Modern Data centre infrastructure

The organization of this paper is as follows, In Section 1 discussed about Introduction about the Traditional Sioled Infrastructure, Section 2 describes the primer SDDC 2.0 converged architecture and its benefits and demerits, Section 3 describes the existing SDDC 3.0 on-premises Hyper-converged Infrastructure to enhance the workloads and resource utilization by means of virtualization, Section 4 elaborates the proposed SDDC 4.0 on-premises composable infrastructure to support modern workloads with container and bare metal to increase scalability in resource utilization. Section 5 gives the conclusion and future directions.

\section{CONVERGED INFRASTRUCTURE 2.0}

To form a Converged Infrastructure (CI) and coupled, integrated model of resources is required and for the easy management of on-premise converged data centre.
The Converged Infrastructure 2.0 helps to increase the efficiency of IT administrator by converging management of CPU, Storage and Network resources. Converged Infrastructure accelerates the IT services, prevents errors and less downtime by means of template-based provisioning [8]. Converged DC is modular, completely pre-configured or pre-architected for increased efficiency called POD's (Performance Optimized Datacenters). CI helps with less compatibility issues by reducing and saving costs for power, cooling, cabling and floor spacing. In CI some components or resources are virtualized with specific tools. As a result, it gives cost effective and dynamic infrastructure without the need to add physical components for the rapidly changing workload requirements day-by-day. CI specific configurations would support specific workloads.

$\mathrm{CI}$ also helps by providing a platform for modular deployment of resources in data centre, repeatable, rapid scalable and for consistent performance. It also reduces the deployment risk by providing or offering "Vendor validated" solutions that speed-up the work with this trusted platform.

\subsection{Deployment methods of Converged Infrastructure:}

The two ways of deploying the converged Infrastructure are: as reference architecture for deploying the converged infrastructure and pre-packed configuration is the another way.

\section{- Pre-Racked configuration:}

This method is for scale-out capability for accelerating faster deployments. Here the Compute, storage, network components are pre-installed in POD rack.

\section{- Reference architecture:}

It has configuration manuals or guidelines that contain a "quick guide", connectivity for CI resources. To leverage the performance of existing equipment this Reference Architecture allows fast and trusted configurations. All the resources like compute, storage and network are deployed according to the vendor guidelines or through Blue Print recommendations. With these pre-configured vendor blue prints, application administrators can be easily scale-up or scale-out the individual components easily [9].

\subsection{Benefits of Converged Infrastructure (CI):}

CI provides single platform system which simplifies the IT operations by means of making them more organized, responsive and efficient. Some of the key benefits of CI are as follows.

\section{- Easy Maintenance}

Since there was less hardware components, the demand for maintenance is reduced as compared to siloed traditional infrastructure. With the capabilities of server centralization, operations and control on resources and applications is easily managed.

\section{- Flexible Scalability}

Through CI, one can scale required the specified components alone for an application that requires for 
execution and the rest of the architecture need not to be disturbed. If the application needs more CPU power, it can be added and then more storage capacity needed by an application it can be commissioned depends on the requirement [19].

\subsection{De-merits of CI:}

- The CI infrastructure contains only storage and server resource components.

- The CPU, storage and Networking ratio of resource components are pre-defined and it can be as flexible as users may need.

- It cannot be suited for existing legacy or prevailing systems.

- $\mathrm{CI}$ is mostly hardware based with less virtualization tools.

- In CI integration would be handled by vendors, user has to still pay for proprietary management and hardware.

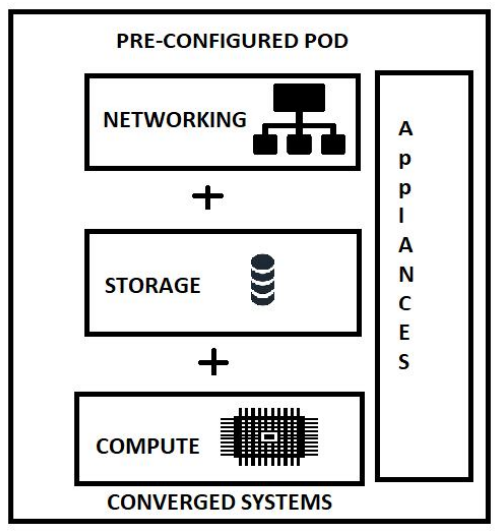

Figure 2: Converged Infrastructure 2.0

In figure 2 attain more flexibility in scaling and to go beyond converged server and storage predefined solutions, Hyper-converged Infrastructure (HCI) has evolved which is purely software-defined datacenter infrastructure to reduce cost over 5 to 70 percentage from legacy stiff systems be eliminating separate storage network.

\section{ON-PREMISES HYPERCONVERGED SDDC INFRASTRUCTURE 3.0}

\subsection{The Emergence of SDDC:}

As the data center keep on evolving there is an emerging need for agility, flexibility, automation, orchestration and control. It is a Software Defined Data Centre (SDDC) a complete virtualized platform built on three pillars with hardware, software and standard interfaces. The abstraction of physical hardware components or resources like CPU, storage and Network through hypervisor and Network Function Virtualization (NFV) software makes a perfect platform for nurturing all future changes and growth in IT industry. This platform enables customer to be flexible and agile in cost optimization. Software-Defined concept makes the on-premises datacenter as true business achiever with high profit. SDDC is a way of expressing a data center where as to split resources into many chunks as possible are abstracted to software. The SDDC is characterized by abstraction of resources through software and code as infrastructure. Through SDDC we can create a new instance with single click and migrate the workload that is running between the physical servers is the overall essence of software-defined approach.

\subsection{Some of the features of SDDC are:}

- Security:

More granular security and compliance through policy based and self-heal solution.

- Flexibility:

Smart Integration with prevailing infrastructure and use of devices of one's own choice.

- Easy Management:

Service portal are user-defined with Application Program Interface (API).

\subsection{HYPERCONVERGED INFRASTRUCURE (HCI) 3.0:}

Today it is possible for virtualizing CPU, storage and network beyond processing and keeps it in a single rack or even in a single individual machine. This type of architecture following in data centers is called hyper-convergence [10]. Hyper meaning quickly or with hypervisor and convergence rapidly coupled together with hypervisor or software. HCI converges the entire data center stack of compute, storage, network and virtualization. HCI replaces the legacy, expensive infrastructure which is complex by a platform runs with end-end systems management, powerful servers that allow medium on-premise enterprises to scale up to one node at a time initially. Software which runs on each host distributes operating functionalities across clusters for agility and resiliency. Today's Hyper-Convergence infrastructure brings storage with compute and in future it may bring into the box with network also or even more. The International Data Corporation predicts the market growth of Hyper-Convergence was $65 \%$ until 2019 with 3.95 billion dollars on sales [11]. HCI is a simplified scalable model which helps to reduce CAPEX (Capital Expenditure) with Operations Expenditure (OPEX)

HCI has gained significant popularity recently as an infrastructure where the resources are clubbed together using virtualization techniques [12]. It is a Virtual Data Centre Environment seamlessly integrates many data center services as an appliance to support various workloads like big data analysis, Customer Relationship Management (CRM) and Enterprise Resource Planning (ERP). HCI architectural model for IT uses X86 based CPU and Hard Disk Drive in a single building block. Hyper-Converged architecture is 
distributed in nature and it connects over IP-based network or with $20 \mathrm{~Gb}$ Ethernet [13]. Using these configuration, it is possible to scale-out high performance clusters to deploy wide range of applications, Hadoop workloads, SQL databases. In HCI storage is of distributed Network Attached Storage (NAS) or Storage Area Network (SAN) which is fault tolerant, storage sharing with each physical node by local caching and thereby connected with high speed network fabrics. An HCI Scale-out option significantly reduces the cost and improves scaling, deploying, managing the storage sub-systems [22].

The Hyper-Converged systems have been provided by Nutanix, HPE, VMware and CISCO. HCI uses REST based interface for automation process in the IT datacenter infrastructure. HCI vendors are at present they are offering their own software stack for various orchestration and control software with open source as well as proprietary architectures. The HCI ensures the transition or evolution of IT infrastructure by commissioning new resources and decommissioning the old obsolete resources by not affecting the services and delivery process.

[14] HCI hardware and software platform configuration by Nutanix is based on various hypervisors such as ESXi Hypervisor which is of Type-I used in data centers for superior virtualization, KVM (Kernel based VM), Hyper-V to deploy any application at any scale in IT infrastructure. To integrate intelligently and reliably with CPU resources, storage, virtualization and network and other management tools into an appliance in an $\mathrm{HCI}$ infrastructure vSAN ReadyNode is used [15]. Two nodes are used for deploying a cluster and expansion with 1-node increment. In HCI each node contains 12 to 24 processor cores and RAM up to 380 GB RAM, 12 TB of HDD, 2 SSD and 10Gb Ethernet port for network communication. In HCI one cluster and scale-out up to 64 hosts and provides $120 \mathrm{VM}$ for a node to the user. This solution will be provided by VMware vSphere 6.7. HCI has moved virtualization and IT strategies to new level by means of transforming the hardware centric to application centric approach which emphasizes and leverages on Software-Defined Infrastructures to deliver "anything as services" like cloud in the on-premise IT environment shown in figure 3 [16] [17].

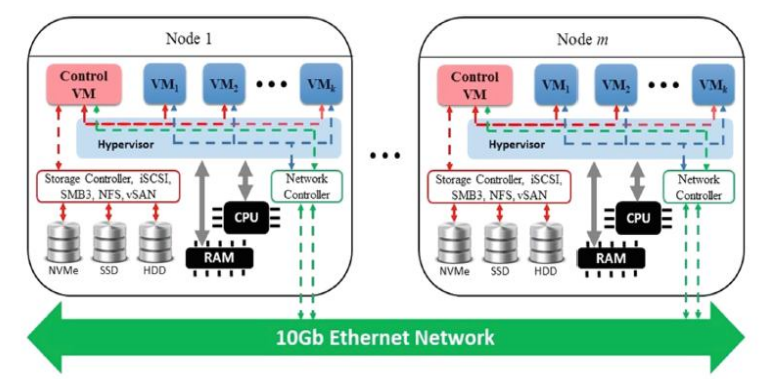

Figure 3: Hyper-Converged Infrastructure 3.0

\subsection{The main components HCI consists of: Distributed plane}

It gives services such as CPU, storage, virtualization and network for the guest applications irrespective of Virtual Machines. This plane runs across a cluster of nodes to provide these services with superior performance [18].

- Control plane or management plane

This will allow for monitoring and controlling easily the HCI resources as single pane view from a place and shirk the need for the separate management for the resources such as CPU, Storage, Storage networks like vSAN and virtualization.

\subsection{Scale-Up vs. Scale-Out Architecture in Hyper- Converged Systems}

\section{- Scale Up Defined Architecture}

With the scale up architecture in hyper convergence solutions you have the choice to add more CPU, Storage, memory or storage throughput to the existing hosts or nodes in the hyper-converged cluster. Scale-up means adding more resources to the server vertically and this will be suits good for on-premise DC enterprises are shown in figure 4.

\section{- Scale Out Defined}

With Scale-out solutions the resources like CPU, storage capacity, memory can be added horizontally that means if you need more capacity of storage another node has to be completely added with more resources leads to over-provisioning. The Hyper-Convergence design uses scale-out design with appliance-approach most of the times it leads to over-provisioning. This Scale-out architecture is also called Linear Scalability.
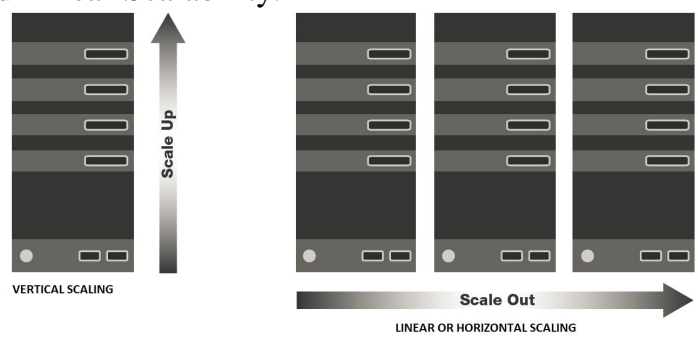

Figure 4: Vertical Vs Linear Scaling in HCI

\subsection{Advantages of Hyper-Converged Infrastructure (HCI):}

\section{Better Scaling}

The Enterprise IT should be more responsive to rapidly adopt the new business needs, setup hardware in some hours and make ready with the workloads in minutes. Make the business critical applications to accelerate their performance by means of HCI to scale up or scale out to meet certain application needs.

\section{- Lower Cost with better storage}

With no disruptions reduce the CAPEX by using X-86 architecture servers for scale up or scale out which is not expensive or deliberate-built networking. With HCI 
Bharani Dharan G et al., International Journal of Advanced Trends in Computer Science and Engineering, 9(4), July - August 2020 , 4964 - 4971

over-provisioning can be avoided and greatly reduced the spending of cost on DC infrastructure.

\section{- Improved IT Efficiency}

Through a single view control plane IT team can now configure, monitor and manage resources and efficient storage capabilities. In HCI since the resources are dynamic, it can be provisioned rapidly, not over-provisioned and with protection.

\section{- Disk-based backup systems}

In HCI these Disk-based backup systems performs instant recovery in virtual machines in proper intervals regularly. So this technique would be used to back up the data during the disasters but the backup platform should have more compute, storage and memory resources that allow the host node to have the copy of Virtual Machine (VM).

\section{- Information Efficiency:}

HCI ensures the high degree of data reduction size by means of de-duplication and compression which gives more storage space, high utilization, more bandwidth and data efficiency.

\section{- Cost:}

HCI helps in reducing the cost of purchasing segments by means of HCI hub from a single seller to deal with customization instead of purchasing the segments or renting from several vendors.

\subsection{De-merits of HCI:}

\section{- Vendor Lock-in}

For Virtual workloads customers rely on single vendor for choosing HCI solution and that single vendor is providing the compute, storage or network. When there is an issue they cannot move to other vendor for providing best balance capabilities in infrastructure or troubleshooting.

\section{- Limitation in Scaling Granularity}

To get additional storage in HCI environment need to add an extra node that also contains compute, network and memory resources. For extra storage no need for these resources. If one can need added compute, additionally storage to the environment with additional node will be added. HCI do not lend itself to scaling granularity for the capacity of specific resources and also leads to over-provisioning.

The new generation of organizations and modern applications focuses on operational velocity with highly customized hardware resources on-demand. Hyper-convergence is not having the capability to focus the mix and match resources on-demand, also HCI supports only virtualization and it is not enough to meet the new modern business demands on the fly. Hence there is a paradigm shift required not only to redesign the physical layer also to to build new methodologies and management capabilities. Hence Composable Infrastructure has evolved to create the perfect blend of eco-system through the modern infrastructure can thrive the IT business enterprise without over-provisioning.

\section{ONPREMISES COMPOSABLE INFRASTRUCTURE (COI) 4.0}

Composable Infrastructure helps to respond IT for new challenges laid down by modern enterprises. COI can be considered as the next phase of Hyper-Convergence infrastructure and next version of on-premise infrastructure. Composable infrastructure consists of fluid pool of multiple resources like CPU, storage and network that makes infrastructure highly dynamic, flexible and capable to run the any kind of workload that is from traditional to modern workloads. COI eliminates the workload-specific environment and avails fluid set of resources to dynamically mix and combine to solve the unique need of application or workloads. COI provides and tweaks best application performance, reduces over provisioning or under provisioning and thereby to create more intelligent, cost-effective and agile data centre. COI enables the on-premises infrastructure to deliver and deploy the applications like on-par cloud services with velocity and simplicity. Based on the given requirement $\mathrm{COI}$ gives the flexibility to compose or re-compose the fluid pools of resources. Through RESTful Unified API calls in the COI we can programmatically automate, control the composition or decomposition of resources with orchestration intelligence by Infrastructure- as-Code ( $\mathrm{IaC})$ near real-time according to the any workload demand by means of prediction techniques using Machine Learning (ML) are shown in figure 5

Software defined Intelligence in COI can be achieved by means of provision, self-discovery, scale-out and auto-integrate. Intelligence in COI can be achieved through implementing Software-Defined approach in life cycle management of hardware components. To create a Software-Defined Infrastructure in COI eco-system should leverage the scalable and flexible development of applications with management tools, blue prints, reference architectures, and automated tools like ansible, chef, puppet etc [23].

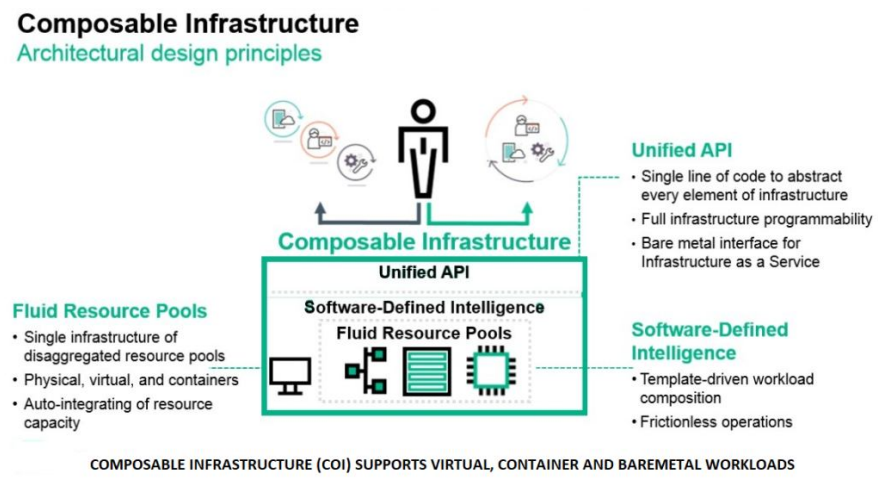

Figure 5: Composable Infrastructure (COI)

\subsection{The Components of Composable Infrastructure}

\section{- Composable Compute}

Composable compute provides flexibility on comparable to available converged or hyper- converged architecture because it provides scalability, flexibility, increased and improved 
Bharani Dharan G et al., International Journal of Advanced Trends in Computer Science and Engineering, 9(4), July - August 2020,4964 - 4971

performance, capacity optimization for container, virtual, bare-metal based workloads.

\section{- Composable Storage}

It provides flexibility to meet the storage demands for the variety of workloads. This module provides fluid pool of storage resources to be consumed by the workloads.

\section{- Composable Fabric:}

It is a multi-fabric high speed network to optimize and share the workloads across the storage and infrastructure. It consolidates the backed Datacenter (DC) connections for compute and extends the connectivity to other modules and avoids the need of top rack switches through intelligent network capabilities.

The following figure 6 depicts how the fluid pool of resources or components can be composed and re-composed based on the workload requirement.

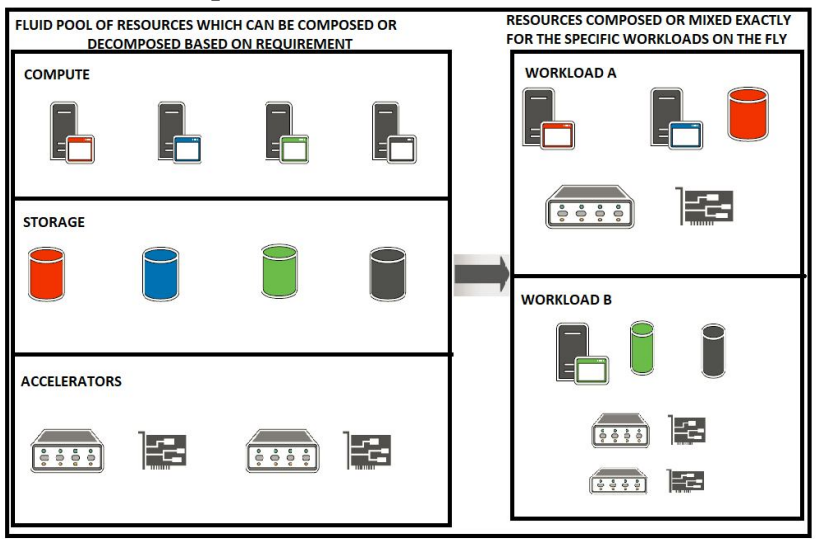

Figure 6: Exact Resources Provisioned from the Fluid Pool for any workloads in COI

\subsection{Orchestration in Composable Infrastructure}

IT Enterprises are becoming more agile by automating and orchestrating their business process. There is always been a confusion arises between automation and orchestration.

\section{- Automation:}

Automation is all about taking a common task ad script them and so that it can be done. Instead of deploying a new window server virtual machine with more clicks, customization and selecting the blueprints from the library rather through scripting it will be deployed in single command by providing the server name with IP address. This single line command will be enabled by scripting. Simply automation is all about automating the tasks.

\section{- Orchestration:}

This works at a high level and leverages the automated tasks. For example, if we want to deploy a 3 tier application with web server, middle-ware server and database server. There is a need of orchestration engine to leverage the automation task. The orchestration combines automated task and automation scripts to deploy a three new window server. Orchestrating is defined simply as it is all about automating the processes [20].

\subsection{Merits of Composable Infrastructure}

- Avoids over- provisioning or under-provisioning

The most problem in the IT sector Data Centre infrastructure is over provisioning or under-provisioning the resources. Under-provisioning will give a pause or make the employees to wait for the resources until the allocation. During the time of workload fluctuation, the over-provisioning may affect the economic growth of an organization may require more investment and it consumes more energy. In this regard COI helps to face the ever-changing needs in Information Technology (IT) to provision the exact resources for any workload from the fluid pools.

\section{- COI for DevOps Teams}

COI helps the DevOps (Development-Operations) team to rapidly create a new environment for launching, deploying the application or workload. The new environment created is with real servers, storage and network but not with few virtual machines. COI leverages the DevOps developers to rapidly compose or recompose the resources.

\section{- COI is for Future-Ready}

Composable Modern Infrastructure provides support for any kind of workload with virtual machines, container or bare metal deployment. It provides right resources at right time for any applications to run. COI provides the potential ability to maintain every element in the Infrastructure by means of Unified API with more scalability and flexibility.

- Dual-way flexibility

In COI we can scale-up and scale-down the resources automatically without any human intervention. The Composability advantage can be achieved without disturbing the business critical applications or workloads.

\section{- Cloud-like speed:}

Composable Infrastructure enables the DevOps teams to deploy or spin up the new services in minutes for deploying the workload faster by cloud-like environment from a secure on-premises DC.

\subsection{De-Merits}

\section{- The software stack}

It is the major problem in the composable infrastructure. In composable infrastructure admins can access and manage resources via API and API's are playing a vital central role to software-driven resource provisioning and it should be open and extensible to the developer to generate code and the resultant code should work with any composable systems located anywhere.

\section{- Lack of Standards for common operation:}

Only CISCO and HPE are actively in the Composable system marketplace and each vendor approach the technology in their own direction will make the resultant product as proprietary. 
In figure 7, the glimpse of paradigm-shift of Datacenter:

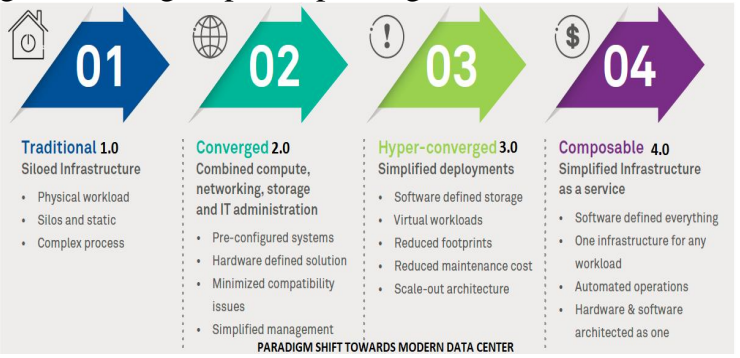

Figure 7: Paradigm shift towards SDDC COI 4.0 [21]

\section{CONCLUSION AND FUTURE DIRECTION}

Composable Infrastructure leverages the dynamic configuration with fluid pool of resources and customization of resources as per specific workloads through right composition that has ensured more profit, cost optimization and better resource utilization, energy saving on comparing the other on-premise architectures. According to the IDC by 202058 percentage of on-premise DC will expand the role to hybrid data centers that are necessary for Next-Gen or cloud-native applications for critical business facility and to meet the modern challenges. In this paper the different on-premises cloud-like or cloud on-par architecture was discussed in the clear sense. In the future work these on-premises cloud like data centers have to be connected and extended with real hybrid-cloud infrastructure to support micro-services based modern applications with resource elasticity, deploying and testing on AWS cloud. On-premises DC can also be extended to hybrid cloud which will provide potential benefits like greater agility, self-service, resiliency, High Availability, cloud bursting solutions and disaster recovery solutions.

\section{REFERENCES}

1. Endo, Patricia Takako, Guto Leoni Santos, Daniel Rosendo, Demis Moacir Gomes, André Moreira, Judith Kelner, Djamel Sadok, Glauco Estácio Gonçalves, and Mozhgan Mahloo. "Minimizing and managing cloud failures." Computer 50, no. 11: pp 86-90, (2017) https://doi.org/10.1109/MC.2017.4041358

2. Challa, Narsimha Reddy. "A cim (common information model) based management model for clouds." In 2012 IEEE International Conference on Cloud Computing in Emerging Markets (CCEM), pp. 1-5. IEEE, 2012.

3. Ferreira, Leylane, Patricia Takako Endo, Daniel Rosendo, Guto Leoni Santos, Demis Gomes, André Luis Cavalcanti Moreira, Glauco Estácio Goncalves et al. "Standardization efforts for traditional data center infrastructure management: the big picture." IEEE Engineering Management Review 48, no. 1: pp 92-103, 2020

4. Ali, Howraa Mehdi Mohammad, Taisir EH El-Gorashi, Ahmed Q. Lawey, and Jaafar MH Elmirghani. "Future energy efficient data centers with disaggregated servers." Journal of Lightwave Technology 35, no. 24 pp: 5361-5380, 2017

5. Katrinis, Kostas, Dimitris Syrivelis, D. Pnevmatikatos, Georgios Zervas, Dimitris Theodoropoulos, Iordanis Koutsopoulos, K. Hasharoni et al. "Rack-scale disaggregated cloud data centers: The dReDBox project vision." In 2016 Design, Automation \& Test in Europe Conference \& Exhibition (DATE), pp. 690-695. IEEE, 2016.

https://doi.org/10.3850/9783981537079_1014

6. Elmirghani, J. M. H., T. Klein, K. Hinton, L. Nonde, A. Q. Lawey, T. E. H. El-Gorashi, M. O. I. Musa, and X. Dong. "GreenTouch GreenMeter core network energy-efficiency improvement measures and optimization." IEEE/OSA Journal of Optical Communications and Networking 10, no. 2 pp: A250-A269, 2018

7. Reese, George. Cloud application architectures: building applications and infrastructure in the cloud. " O'Reilly Media, Inc.", 2009.

8. Azeem, Shaikh Abdul, and Satyendra Kumar Sharma. "Study of converged infrastructure \& hyper converge infrastructre as future of data centre." International Journal of Advanced Research in Computer Science 8, no. 5 (2017).

9. Converged Infrastructure, available at: https://www.netapp.com/us/info/what-is-converged-infr structure.aspx

10. Melo, Carlos, Jamilson Dantas, Andre Oliveira, Danilo Oliveira, Iure Fé, Jean Araujo, Rubens Matos, and Paulo Maciel. "Availability models for hyper-converged cloud computing infrastructures." In 2018 Annual IEEE International Systems Conference (SysCon), pp. 1-7. IEEE, 2018.

11. Worldwide Hyperconverged Systems 2015-2019 Forecast. Available at https://www.marketresearch.com/IDC-v2477/Worldwid e-Hyperconverged-Systems-Forecast-9140365/.

12. Asyabi, Esmail, and Mohsen Sharifi. "New Approach for Dynamic Virtual Machine Consolidation in Cloud Data Centers." International Journal of Modern Education \& Computer Science 7, no. 42015.

13. Rolik, Oleksandr, Sergii Telenyk, and Eduard Zharikov. "IoT and cloud computing: the architecture of microcloud-based IoT infrastructure management system." In Securing the Internet of Things: Concepts, Methodologies, Tools, and Applications, pp. 1157-1185. IGI Global, 2020.

14. Hardware Platforms Explore the range of platforms from Nutanix, our OEM partners and third-party server vendors available at: https://www.nutanix.com/products/hardware-platforms

15. Hyper-Converged Infrastructure. available at: https://www.nutanix.com/hyperconverged-infrastructure

16. Risdianto, Aris Cahyadi, Muhammad Usman, and JongWon Kim. "SmartX Box: Virtualized Hyper-Converged Resources for Building an 
Affordable Playground." Electronics 8, no. 11 pp: 1242,2019

https://doi.org/10.3390/electronics8111242

17. Rolik, Oleksandr, Sergii Telenyk, and Eduard Zharikov. "Management of services of a hyperconverged infrastructure using the coordinator." In International Conference on Computer Science, Engineering and Education Applications,. Springer, Cham, pp. 456-467, 2018.

18. Prism Pro: Streamline Your Infrastructure operations available at: https://www.nutanix.com/products/prism/pro

19. Why Is Betting Big on Composable Infrastructure with synergy systems: available at: https://bwcio.businessworld.in/article/why-Is-Betting-Bi g-on-Composable-Infrastructure-with-synergy-systems-/ 29-03-2016-110808

20. Scott D. Lowe, James Green and David Davis, "Building a Modern Data Center", Atlantis, Published by Published by ActualTech Media In Partnership with Atlantis Computing, ActualTech Media Okatie Village Ste 103-157 Bluffton, SC 29909 USA.

21. Sreenath.M, Composable Infrastructure available at Whitepaper, WIPRO Technologies, APRIL 2020.

22. Indrajani Sutedja, Yaya Heryadi, Lili Ayu Wulandhari, Bachtiar Abbas, "Imbalanced Data Classification Using Auxiliary Classifier Generative Adversarial Networks", International Journal of Advanced Trends in Computer Science and Engineering, Vol 9, No 2, Mar -Apr 2020, pp: 1068-1075, 2020

https://doi.org/10.30534/ijatcse/2020/26922020

23. Muslihah Wook, Zam Zarina Abdul Jabar, Muhammad Hakiem Halim , Noor Afiza Mat Razali, Suzaimah Ramli , Nor Asiakin Hasbullah, et al "Big Data Analytics Application Model Based on Data Quality Dimensions and Big Data Traits in Public Sector", Vol 9, No 2, Mar -Apr 2020, pp: 1247-1256, 2020 https://doi.org/10.30534/ijatcse/2020/53922020 